\title{
5 Demographische Effekte der Studierenden Wiens auf die Stadt Wien
}

\subsection{Die Studierenden Wiens im Überblick}

Gemäß der uni:data-Statistik des Bundesministeriums für Wissenschaft und Forschung waren im Wintersemester 2012 insgesamt 186.990 Studierende an Hochschulen in Wien inskribiert. Dieser hohen Zahl liegt ein beträchtliches Wachstum in den vorangegangenen Jahren zugrunde. Seit dem Wintersemester 2005 hat die Zahl der Studierenden um 52.657 zugenommen, dies entsprach einem Anstieg von 39,2 \% (vgl. Tabelle 5.1). Hinter dieser Entwicklung liegen, neben dem allgemeinen verstärkten Interesse an einem Studium, zwei Trends: Erstens haben sich die rechtlich-finanziellen Rahmenbedingungen für die Studierenden geändert: Mit der Einführung von Studiengebühren im Wintersemester 2001 ist es zu einem einmaligen Rückgang der Studierendenzahl an den Universitäten gekommen, mit deren Abschaffung im Wintersemester 2009 erfolgte wieder ein einmaliger Anstieg, der sich in der Statistik deutlich abzeichnet (siehe auch Abbildung 5.1).

Tabelle 5.1: Entwicklung der Studierendenzahlen in Wien sowie Anteil der Studierenden mit ausländischer Staatsbürgerschaft

\begin{tabular}{lccc}
\hline Semester & Studierende Gesamt & $\begin{array}{c}\text { Veränderung gegenüber } \\
\text { dem Vorjahr (\%) }\end{array}$ & Anteil Ausländer (\%) \\
\hline WS ‘05 & 134.333 & & 18,6 \\
WS ‘06 & 142.395 & 6,0 & 20,0 \\
WS ‘07 & 149.584 & 5,0 & 20,9 \\
WS ‘08 & 154.029 & 3,0 & 22,1 \\
WS ‘09 & 174.088 & 13,0 & 22,7 \\
WS ‘10 & 179.739 & 3,2 & 23,9 \\
WS ‘11 & 184.755 & 2,8 & 25,2 \\
WS ‘12 & 186.990 & 1,2 & 26,9 \\
\hline
\end{tabular}

Quelle: uni:data 2013.

Weiters ist das Wachstum der Studierendenzahlen - neben der Zunahme inländischer Hörer - auch auf einen überproportionalen Anstieg der ausländischen Studierenden zurückzuführen. An den Wiener Universitäten besaß im Wintersemester (WS) 2012 mehr als ein Viertel der Studierenden (27,6 \%) keine österreichische Staatsbürgerschaft, seit dem WS 2005 hat sich deren Zahl beinahe verdoppelt (vgl. Tabelle 5.2). Auch an den Fachhochschulen ist es zu einem überproportionalen Anstieg der ausländischen Studierenden gekommen, wenngleich das Internationalisierungsniveau mit 12,1 
\% im WS 2012 deutlich geringer war als bei den Universitäten. Die Privatuniversitäten in Wien weisen eine traditionell hohe Quote ausländischer Studierender auf. Aktuell machen diese mehr als die Hälfte (52,9 \%) der Studierenden aus, wenngleich dieser Anteil auch seit dem WS 2005 weniger stark wuchs als die Zahl inländischer Studierender. In Summe weist der Trend auf eine stark steigende Internationalisierung des Wiener Hochschulstandortes hin; auf das räumliche Muster dieser ,internationalen Reichweite" wird in Kapitel 5.3 eingegangen.

Die Statistik zeigt des Weiteren, dass die Entwicklung der Zahl der ausländischen Studierenden an den Wiener Hochschulen durchaus einem landesweiten Trend entspricht. So sind etwa die Wiener Universitäten nur geringfügig stärker internationalisiert als die österreichischen Universitäten insgesamt (27,6 \% vs. 26,2 \%, vgl. Tabelle 5.2). Auch bei den Fachhochschulen liegt der vergleichsweise geringe Anteil ausländischer Studierender im landesweiten Trend, während zwischen den Privatuniversitäten deutliche Unterschiede existieren, die auf das unterschiedliche Studienangebot der einzelnen Einrichtungen zurückzuführen sind.

Das WS 2005 stellt für die Internationalisierung der Wiener Hochschulen insofern eine Trendwende dar, als seit diesem Semester die Zahl ausländischer Studierender stärker wächst als die der Inländer, während die Dynamik in den Jahren davor noch eher ausgeglichen war (vgl. Abbildung 5.1). Hintergrund der Entwicklung ist einerseits das Angebot an Mobilitätsprogrammen (etwa Erasmus), die durch die Bologna-Reform verstärkt in Anspruch genommen werden. Darüber hinaus haben auch ausländische Studierende aus Drittstaaten (sogenannten „Freemover") sowie in Österreich geborene Ausländer einen wichtigen Anteil an dem überproportionalen Anstieg ausländischer Studierender. Insgesamt zeigt diese Entwicklung, dass die Wiener Hochschulen eine starke Anziehungskraft auf hochqualifizierte Migrantinnen und Migranten ausüben und damit einen positiven Beitrag zum Migrationsregime der Stadt leisten.

Tabelle 5.2: Studierende nach Hochschultyp (WS 2012) in Wien und Österreich und ausländische Studierende

\begin{tabular}{llrcccc}
\hline & & \multicolumn{2}{c}{ Studierende gesamt } & \multicolumn{3}{c}{ ausl. Studierende } \\
& & absolut & $\begin{array}{c}\text { Veränderung } \\
\text { 2005-2012 (\%) }\end{array}$ & absolut & $\begin{array}{c}\text { Veränderung } \\
\text { 2005-2012 (\%) }\end{array}$ & $\begin{array}{c}\text { Anteil ausl. } \\
\text { Studierende } \\
\text { 2012 (\%) }\end{array}$ \\
\hline \multirow{2}{*}{ Universitäten } & Wien & 171.534 & 36,5 & 47.330 & 97,4 & 27,6 \\
& Österreich & 299.435 & 37,6 & 78.390 & 80,9 & 26,2 \\
\multirow{5}{*}{ Fachhochschulen } & Wien & 12.653 & 78,6 & 1.533 & 344,3 & 12,1 \\
& Österreich & 41.366 & 60,8 & 5.885 & 229,1 & 14,2 \\
\multirow{2}{*}{ Privatuniversitäten } & Wien & 2.803 & 75,1 & 1.484 & 38,2 & 52,9 \\
& Österreich & 7.316 & 102,8 & 2.873 & 167,5 & 39,3 \\
\hline
\end{tabular}

Quelle: uni:data 2013. 
Abbildung 5.1: Index der Studierendenzahl (2005 = 100) von In- und Ausländern an Universitäten, Fachhochschulen und Privatuniversitäten in Wien

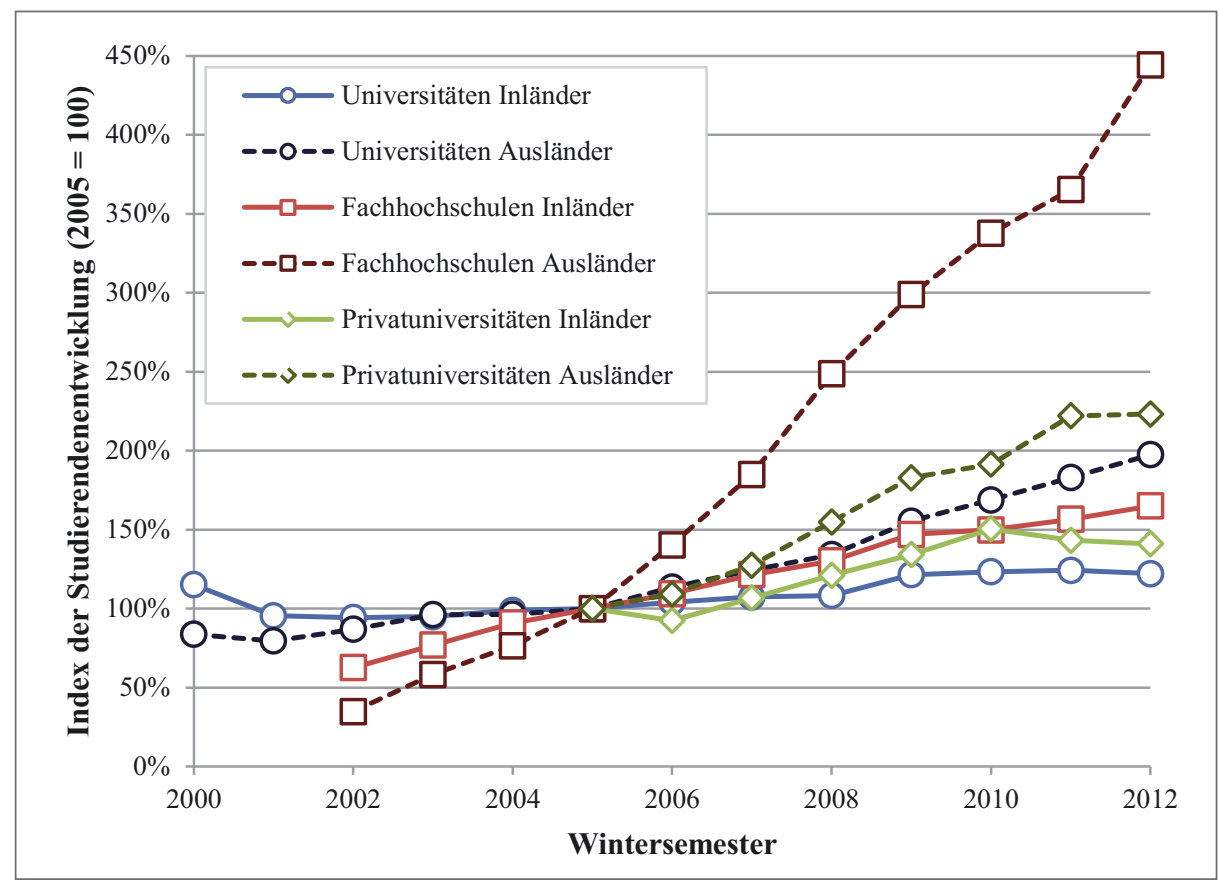

Quelle: uni:data 2013; eigene Berechnungen.

\subsection{Woher kommen die Wiener Studierenden? Wiener, Suburbaniten, „Zuagraste“}

Die Studierenden an den Wiener Hochschulen machen einen nicht unbeträchtlichen Teil der Wiener Stadtbevölkerung aus. Gemessen an der Gesamtbevölkerung Wiens im Jahr 2011 ist beinahe jeder Zehnte (10,7 \%) an einer der tertiären Bildungseinrichtungen in Wien inskribiert (vgl. Tabelle 5.4). Dieses Verhältnis ist angesichts der Größe der Stadt - aktuell nach Berlin immerhin die zweitgrößte Stadt des deutschen Sprachraumes - bemerkenswert und widerlegt damit für Wien die These, dass die Universitäten in den wirklich großen Städten ,versinken“ (van der Wusten 1998, 4). Allerdings weist diese Verhältniszahl auch gewisse Unschärfen auf: Erstens sind viele der Studierenden nicht in Wien, sondern in den Heimatgemeinden gemeldet, was zu einer Reduktion der Grundgesamtheit „Stadtbevölkerung“ führt. Zweitens lebt ein Teil der Studierenden nicht in Wien, sondern pendelt aus dem Wiener Umland an die jeweilige Bildungseinrichtung. Während die erste Fehlerquelle, die zu einer leichten Reduktion der Grund- 
gesamtheit führt, nur durch eine direkte Studierendenbefragung erhoben und damit im Rahmen dieser Studie nicht behandelt werden kann, lässt sich das Ausmaß des ,,Suburbanisierungsgrads" der Studierenden sehr wohl berücksichtigen. Immerhin leben gemäß Stadtregionsabgrenzung der Statistik Austria gegenwärtig 20,9 \% der Bevölkerung der Stadtregion Wien außerhalb der administrativen Grenzen der Kernstadt (Statistik Austria 2013). Die Berücksichtigung dieses Phänomens ist für diese Studie insofern relevant, als die im Wiener Umland lebenden Studierenden durch ihre Ausgaben einen anderen Wertschöpfungseffekt für Wien haben als jene, die innerhalb der Kernstadt leben.

\section{Die Heimatadressen der Studierenden}

Um den demographischen Effekt der Wiener Hochschulen für Wien besser abschätzen zu können, wurden in einer Sonderauswertung die Studierenden nach ihrer Herkunftsregion innerhalb Österreichs differenziert. Grundlage dafür bildet die „Heimatadresse“, die Studierende bei ihrer Immatrikulation angeben müssen und die von dem aktuellen Wohnort abweichen kann. Auch ausländische Studierende sind in diesem Sample enthalten, da auch diese eine „Heimatadresse“ innerhalb Österreichs angeben müssen. Auf Basis dieser Erhebung ist es für die Hochschulen, die Daten zur Verfügung gestellt haben, möglich, ein präzises Bild des räumlichen Einzugsgebietes nachzuzeichnen. Für die Wertschöpfungseffekte wichtiger ist vor allem der Anteil jener Studierenden, der nicht in der Kernstadt, sondern im Wiener Umland lebt. Deshalb wurden die Studierenden nach drei Regionen differenziert:

- $\quad$ Studierende mit Heimatadresse Wien, d.h. jene, die am Wohnort Wien studieren.

- Studierende mit Heimatadresse im Wiener Umland, die zwischen Wohn- und Studienort pendeln.

- Studierende mit Heimatadresse außerhalb des Wiener Umlandes, denen aufgrund der unzumutbaren Pendlerdistanz unterstellt wird, in Wien zu wohnen (also Wochen-/Monatspendler bzw. Zugewanderte).

Bei der Definition des Wiener Umlandes, ein zentrales Thema der Stadtgeographie (vgl. Fassmann 2009), wurde nicht auf die Stadtregionsabgrenzung der Statistik Austria zurückgegriffen, da sich diese unter anderem an den Pendlerbeziehungen orientiert und neben Wien auch andere Einpendlerzentren (etwa Mödling, Baden usw.) berücksichtigt (vgl. Wonka und Laburda 2010). Um dem spezifischen Mobilitätsverhalten der Studierenden sowie der Ausrichtung auf den Standort Wien gerecht zu werden, wurde das Wiener Umland auf Grundlage der Erreichbarkeitsverhältnisse des öffentlichen Personennahverkehrs (ÖPNV) abgegrenzt (vgl. Karte 5.1). Dabei wurden all jene Gemeinden zum Wiener Umland gezählt, von denen aus ein hochrangiger Pendlerknoten innerhalb Wiens in einer durchschnittlichen ÖPNV-Reisezeit von 45 Minuten erreicht werden kann. Demnach zählen 219 niederösterreichische und 11 burgenländische Gemeinden zum Wiener Umland. 
Karte 5.1: Abgrenzung des Wiener Umlands auf Basis der Erreichbarkeitszeiten von Pendlerknotenpunkten mit dem ÖPNV in Wien

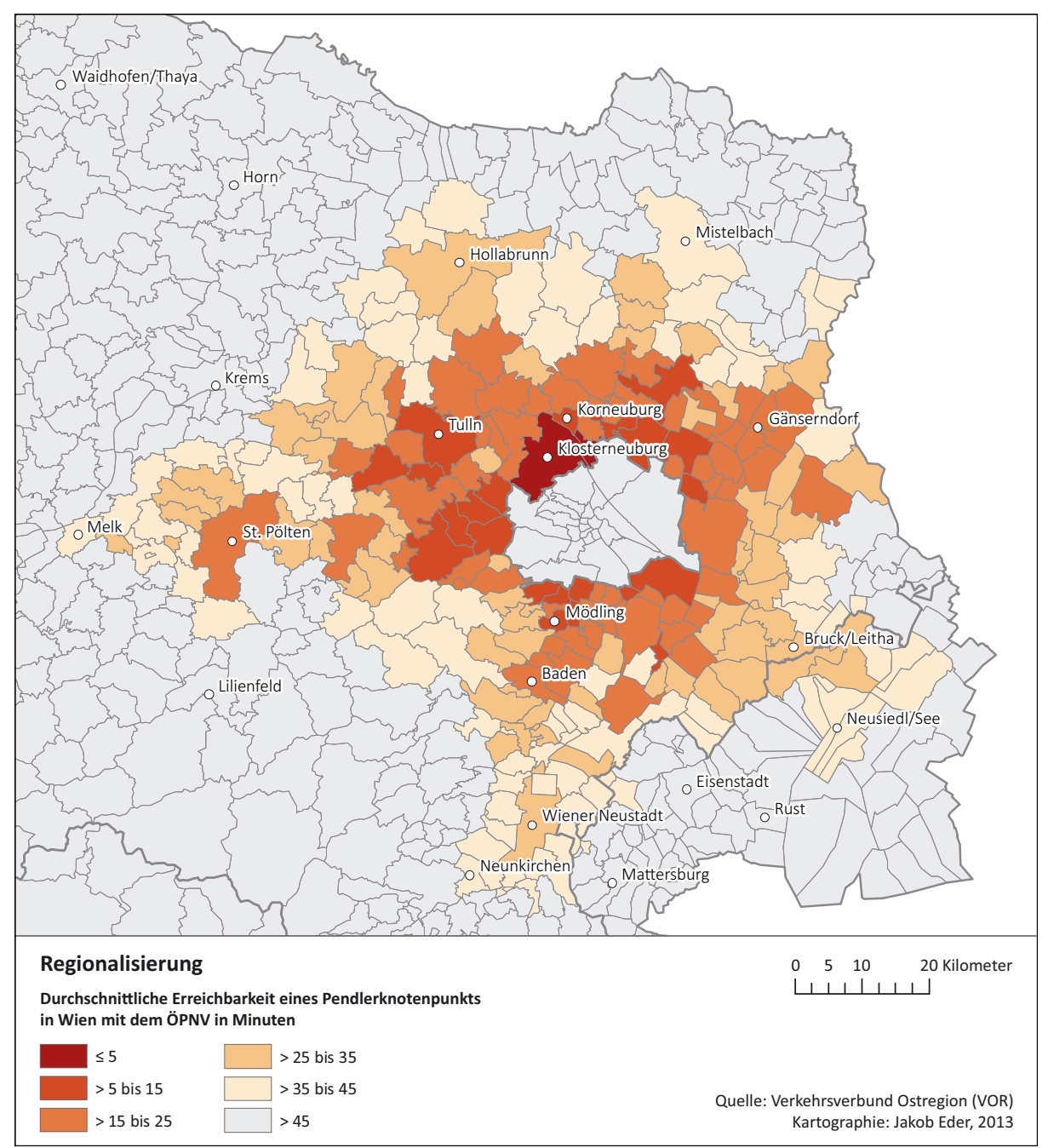

Die Verortung der Studierenden nach der Heimatadresse konnte nicht für alle Hochschulen durchgeführt werden, überdies bestehen auch bei den ausgewerteten Einrichtungen Erhebungslücken. Für die Universität Wien wurden beispielsweise 90,1 \% der Studierenden regional (auf Gemeindeebene) verortet. Aus diesem Grund wurde die Verhältniszahl Stadt-Umland-Rest zuerst auf die Gesamtzahl der Studierenden in den jeweiligen Hochschulen sowie anschließend auf die restlichen Hochschulen, die nicht 
ausgewertet wurden, hochgerechnet. Auch die Verteilung der Studierenden nach Bundesländern wurde auf diese Weise geschätzt.

Die Verortung der Studierenden nach der Heimatadresse hat ergeben, dass 60,4 \% aller Studierenden Wien als Heimatadresse angegeben haben, wobei die Tendenz gegenüber den beiden Vorjahren leicht steigend ist (vgl. Tabelle 5.3). Weiters haben 27.396 Studierende eine Gemeinde im Wiener Umland als Heimatgemeinde angegeben, womit der Anteil der „suburbanen“ Studierenden 14,9 \% ausmacht. Diese Gruppe pendelt täglich an den Studienort in Wien. Umgekehrt ergibt sich daraus, dass 85,1\% der Studierenden in Wien leben, also neben jenen, die Wien als Heimatadresse angegeben haben, sind auch jene 45.691 Studierenden hinzuzuzählen, deren Heimatadresse nicht in einer zumutbaren Pendlerdistanz zu Wien liegt. Damit reduziert sich die Zahl der in Wien lebenden - und für die Wertschöpfung relevanten - Studierenden auf 157.359, womit diese um die einpendelnden Studierenden bereinigt 9,1 \% der Wiener Gesamtbevölkerung ausmachen (vgl. Tabelle 5.4). Trotz der Größe der Stadt ein noch immer beträchtlicher Wert.

Die regionale Differenzierung nach Bundesländern zeigt die Dominanz des östlichen Österreichs im Einzugsgebiet des Hochschulstandortes Wien (vgl. Tabelle 5.3); 85,2 \% der in Wien Studierenden haben eine Heimatadresse in Wien, Niederösterreich oder dem Burgenland angegeben. Aus den restlichen sechs Bundesländern sticht Oberösterreich hervor; immerhin stammen von den rund 27.300 Studierenden aus den west-

Tabelle 5.3: Heimatadresse der Studierenden Wiens nach Bundesländern und Stadtumland

\begin{tabular}{lrrrc}
\hline Bundesland & 2009 & 2010 & 2011 & Anteil 2011 (\%) \\
\hline Wien & 104.206 & 108.644 & 111.668 & 60,4 \\
Niederösterreich & 37.014 & 37.988 & 38.970 & 21,1 \\
$\quad$ davon Umland Wien & 24.874 & 25.617 & 26.357 & 14,3 \\
$\quad$ davon restliches Niederösterreich & 12.139 & 12.371 & 12.613 & 6,8 \\
Burgenland & 6.554 & 6.633 & 6.811 & 3,7 \\
$\quad$ davon Umland Wien & 925 & 950 & 1.039 & 0,6 \\
$\quad$ davon restliches Burgenland & 5.629 & 5.683 & 5.772 & 3,1 \\
Oberösterreich & 11.801 & 11.877 & 12.455 & 6,7 \\
Steiermark & 4.259 & 4.242 & 4.240 & 2,3 \\
Kärnten & 3.496 & 3.451 & 3.543 & 1,9 \\
Vorarlberg & 2.642 & 2.642 & 2.699 & 1,5 \\
Salzburg & 2.511 & 2.581 & 2.650 & 1,4 \\
Tirol & 1.604 & 1.679 & 1.719 & 0,9 \\
\hline Gesamt & $\mathbf{1 7 4 . 0 8 8}$ & $\mathbf{1 7 9 . 7 3 9}$ & $\mathbf{1 8 4 . 7 5 5}$ & $\mathbf{1 0 0 , 0}$ \\
\hline
\end{tabular}

Quellen: eigene Erhebung 2013; uni:data 2013; eigene Berechnungen. 
Tabelle 5.4: Verhältnis der in Wien lebenden Studierenden zur Gesamtbevölkerung der Stadt Wien

\begin{tabular}{lrrr}
\hline & \multicolumn{1}{c}{2009} & \multicolumn{1}{c}{2010} & \multicolumn{1}{c}{2011} \\
\hline Bevölkerungszahl Wien & 1.693 .047 & 1.706 .482 & 1.722 .689 \\
Studierende Wien & 174.088 & 179.739 & 184.755 \\
Anteil & $10,3 \%$ & $10,5 \%$ & $10,7 \%$ \\
\hline \hline Studierende bereinigt & 148.289 & 153.171 & 157.359 \\
Anteil bereinigt & $8,8 \%$ & $9,0 \%$ & $9,1 \%$ \\
\hline
\end{tabular}

Quellen: eigene Erhebung 2013; uni:data 2013; Stadt Wien 2013; eigene Berechnungen.

lichen und südlichen Bundesländern etwas weniger als die Hälfte (12.455) aus Oberösterreich. Für das räumliche Muster des Einzugsgebietes zeigen sich auch Unterschiede zwischen den Hochschultypen: Der Anteil der in der Suburbia lebenden Studierenden ist bei den Universitäten spürbar geringer als bei den Fachhochschulen, von denen knapp jeder Fünfte (19,1 \%) in der Wiener Suburbia lebt. Der Anteil von Studierenden mit einer Heimatadresse im restlichen Österreich ist bei den Privatuniversitäten am geringsten. Das grobe räumliche Muster - die starke Konzentration auf die Stadtregion Wien - zeichnet sich hingegen bei allen drei Hochschultypen ab (vgl. Abbildung 5.2).

Abbildung 5.2: Heimatadresse der Studierenden Wiens insgesamt und nach Hochschultyp

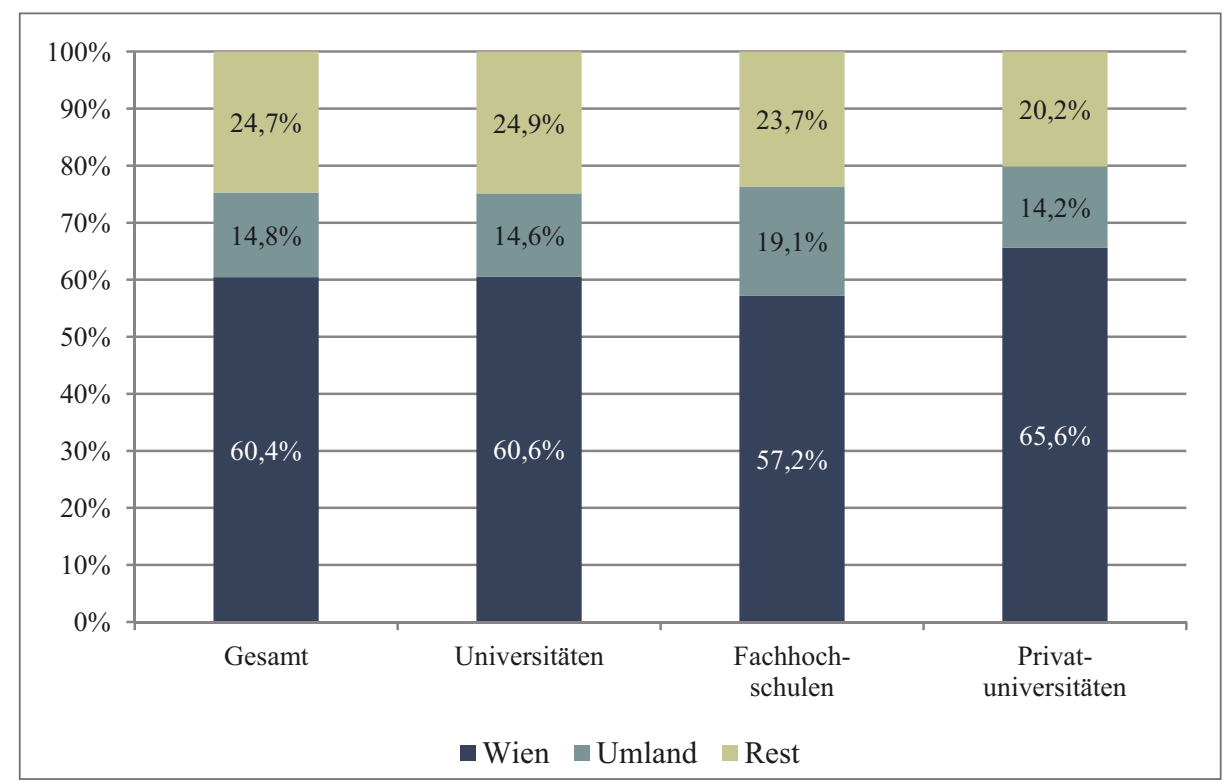

Quelle: eigene Erhebung 2013; uni:data 2013; eigene Berechnungen. 
Obwohl die österreichische Ostregion als Herkunftsregion der Studierenden sehr deutlich dominiert, zeigt sich in der regionalen Analyse, dass der Hochschulstandort Wien ein geschlossenes und weit gestreutes Einzugsgebiet hat - aus jedem Politischen Bezirk in Österreich sind Studierende an einer Wiener Hochschule inskribiert. Das räumliche Muster zeigt auch hier das ausgeprägte West-Ost-Gefälle in etwas differenzierter Form (vgl. Karten 5.2 und 5.3): Abgesehen vom nahen Wiener Umland scheinen die Erreichbarkeitsgefälle eine wichtigen Einfluss zu haben: Bezirke entlang der Westund Südbahnachse spielen eine wichtigere Rolle als solche in peripheren Regionen. Interessanterweise stammen im Fall zahlreicher Landeshauptstädte (Innsbruck, Graz, Salzburg, Klagenfurt) mehr Studierende aus den Umlandbezirken als aus der Landeshauptstadt selbst. Womöglich liegt dies am attraktiveren Bildungsangebot ,vor Ort“ in den Landeshauptstädten, das einen Umzug nach Wien weniger interessant macht. Bei Fachhochschulen zeigt die regionale Streuung nur insofern Unterschiede, als dass die Ostregion als Einzugsgebiet noch bedeutender ist (vgl. Karte 5.3).

Eine stärkere Differenzierung ergibt sich hingegen, wenn man die räumlichen Einzugsgebiete einzelner Universitäten oder Fachhochschulen vergleicht. So besteht bei der Universität für Veterinärmedizin oder der Universität für Bodenkultur aufgrund der Singularität ihrer in Österreich angebotenen Studienrichtungen ein hochgradig disper-

Karte 5.2: Herkunft der Studierenden der Universitäten Wiens nach Politischen Bezirken

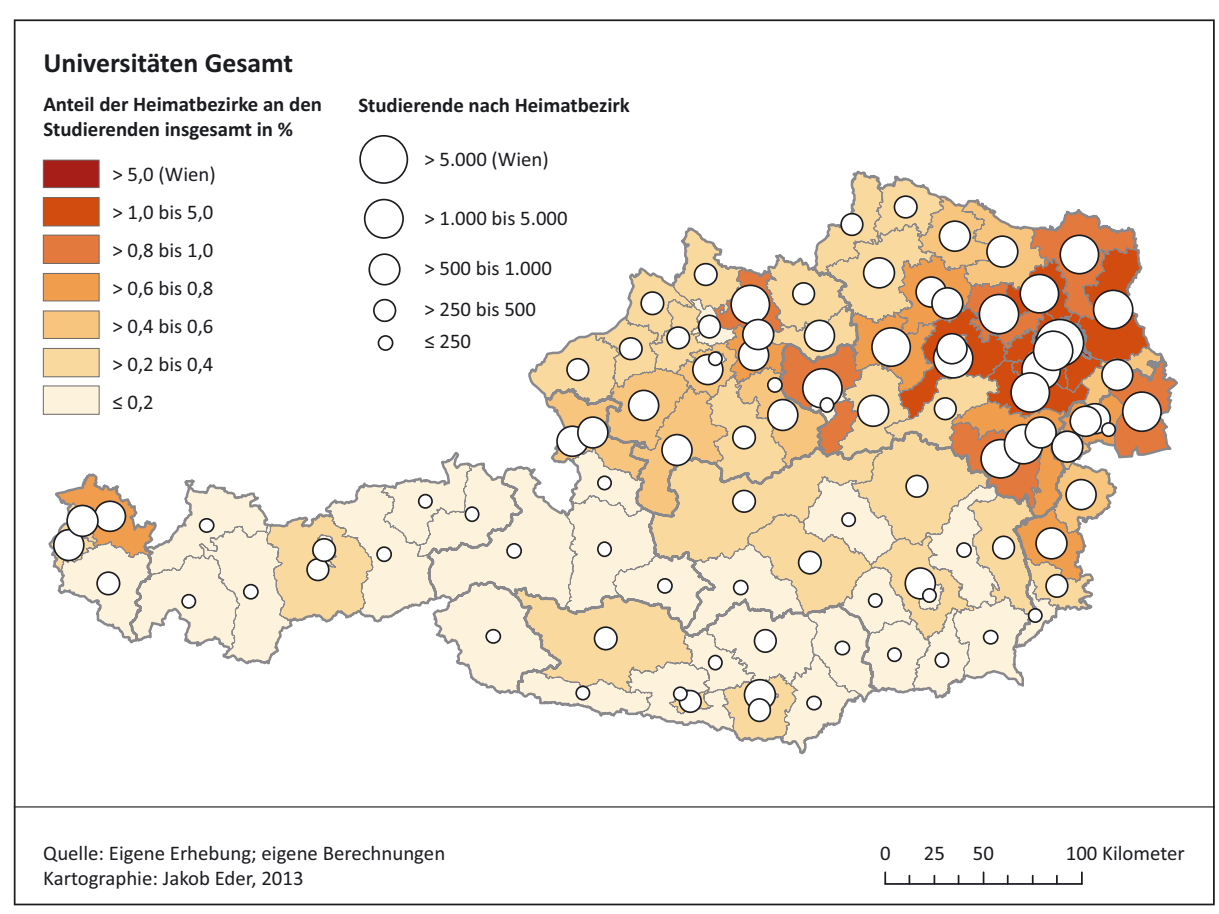


ses Einzugsgebiet, während die Universität Wien zwar nicht gerade über ein ubiquitäres, aber doch breites Studienangebot verfügt, das auch an mehreren anderen Standorten angeboten wird (siehe Anhang I).

Karte 5.3: Herkunft der Studierenden der Fachhochschulen Wiens nach Politischen Bezirken

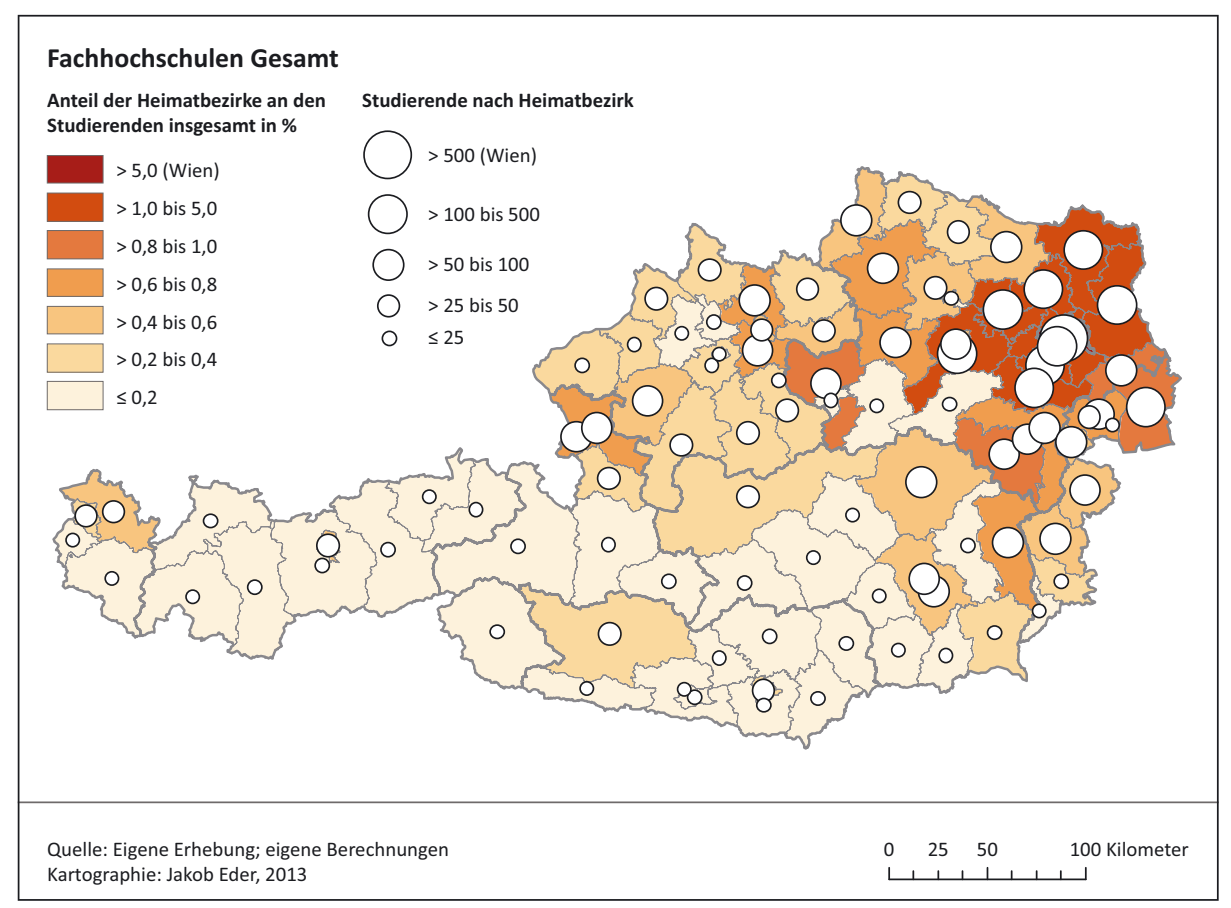

\subsection{Staatsbürgerschaft der ausländischen Studierenden}

Internationale Studierende haben als hochqualifizierte Migranten einen wichtigen Einfluss auf einen Universitätsstandort, der über die Frage der direkten monetären Wertschöpfung hinausgeht. Sie tragen zur Internationalität der Hochschulen bei und fördern den Brain-Gain Hochqualifizierter, da Studierende aus persönlichen oder beruflichen Gründen nach Studienabschluss den Studienort häufig nicht mehr verlassen. Das Ausmaß, in dem Universitäten in der Lage sind, ausländische Studierende anzuziehen, ist einerseits Ausdruck der Wettbewerbsfähigkeit und des Images der Hochschulen, andererseits auch der Lebensqualität und des Images des jeweiligen Standortes. Innerhalb der Europäischen Hochschullandschaft mit ihrem ausgeprägten West-Ost- und NordSüd-Gefälle nimmt Wien eine Zwischenstellung ein: zwar zeigt sich bei den ERAS- 
MUS-Studierenden eine negative Bilanz (vgl. Abbildung 5.4), jedoch zieht Wien eine beträchtliche Zahl an Freemovern, neben dem deutschsprachigen Ausland vor allem aus dem ost- und südosteuropäischen Raum, an. Vor diesem Hintergrund ist es naheliegend, dass Hochschulen eigene Internationalisierungsstrategien entwickelt haben, um ein internationales Profil in Forschung und Lehre (weiter-)zuentwickeln (vgl. Universität Wien 2011, 4). Auch die im europäischen Vergleich sehr hohe Lebensqualität Wiens für

Karte 5.4: Europäische Hochschulstandorte auf Basis der Bilanz der ERASMUS-

Studierenden (incoming/outgoing)

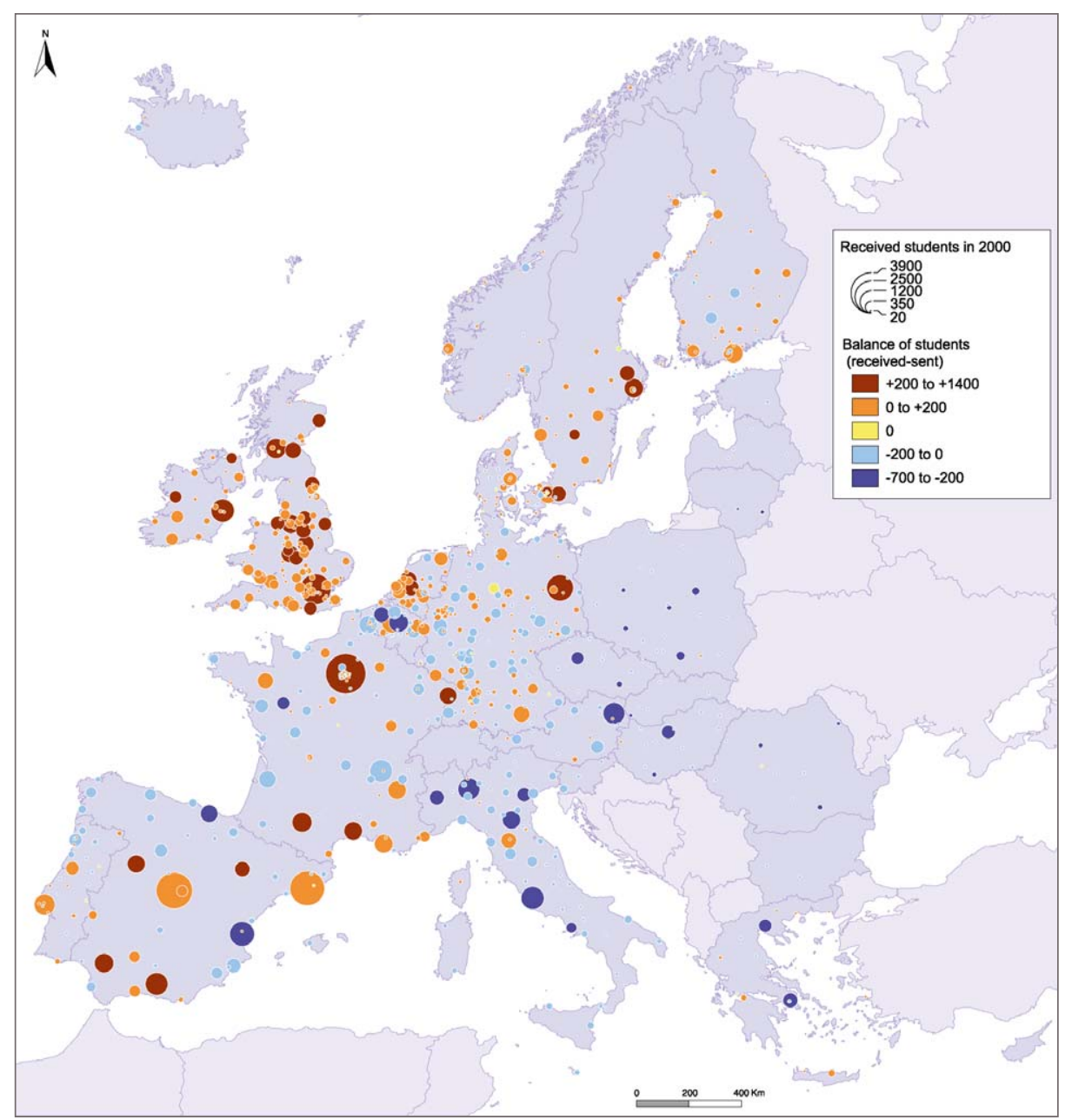

Quelle: Rozenblat und Cicille, 2003. 
Studierende ${ }^{1}$ unterstreicht die Bedeutung der Stadt in der europäischen Hochschullandschaft, wenn sie auch nicht unbedingt zu dessen Zentrum zählt.

Die Motive für die Wahl eines Studienorts - sowohl studienbezogene wie nicht-studienbezogene - variieren sehr stark nach dem Herkunftsland der Studierenden, wie Kohlbacher und Reeger (2005, 64-69) in einer Erhebung zum Studienstandort Wien feststellen konnten. Der Einzugsbereich der Hochschulen für ausländische Studierende (Studierende mit nichtösterreichischer Staatsbürgerschaft) wird von unterschiedlichen Faktoren beeinflusst: einerseits der gemeinsamen (deutschen) Sprache, den rechtlichen Rahmenbedingungen des Zuganges, der Herkunft von im Land lebenden Migranten ohne österreichische Staatsbürgerschaft sowie vom Image von Universität und Stadt im Ausland.

Tabelle 5.5: Herkunft der ausländischen Studierenden Wiens nach Kontinenten/Weltregionen für alle Hochschulen (WS 2009 bis WS 2011)

\begin{tabular}{|c|c|c|c|c|c|}
\hline & Anzahl Staaten & 2009 & 2010 & 2011 & Anteil $2011(\%)$ \\
\hline Europa & 40 & 38.122 & 34.936 & 38.122 & 81,9 \\
\hline Osteuropa & 11 & 9.966 & 9.282 & 9.966 & 21,4 \\
\hline Nordeuropa & 10 & 1.115 & 982 & 1.115 & 2,4 \\
\hline Südeuropa & 12 & 8.376 & 7.936 & 8.376 & 18,0 \\
\hline Westeuropa & 7 & 18.665 & 16.736 & 18.665 & 40,1 \\
\hline Afrika & 41 & 627 & 636 & 627 & 1,3 \\
\hline Nord- und Westafrika & 19 & 414 & 428 & 414 & 0,9 \\
\hline Zentral-, Ost- und Südafrika & 22 & 214 & 208 & 214 & 0,5 \\
\hline Amerika & 24 & 1.129 & 1.008 & 1.129 & 2,4 \\
\hline Nordamerika & 2 & 521 & 473 & 521 & 1,1 \\
\hline Mittelamerika und Karibik & 12 & 118 & 99 & 118 & 0,3 \\
\hline Südamerika & 10 & 490 & 437 & 490 & 1,1 \\
\hline Asien & 46 & 6.569 & 6.081 & 6.569 & 14,1 \\
\hline Zentralasien & 5 & 232 & 182 & 232 & 0,5 \\
\hline Westasien & 17 & 3.712 & 3.437 & 3.712 & 8,0 \\
\hline Süd-, Ost- und Südostasien & 24 & 2.626 & 2.462 & 2.626 & 5,6 \\
\hline Ozeanien & 2 & 109 & 115 & 109 & 0,2 \\
\hline Gesamt & 153 & 39.232 & 42.958 & 46.558 & 100,0 \\
\hline
\end{tabular}

Quellen: eigene Erhebung 2013; eigene Berechnungen.

1 Wien liegt im „Best Student City Ranking“ 2012 in Europa hinter London und Paris auf Rang 3, weltweit auf Rang 5 (www.topuniversities.com/city-rankings/2012; Zugriff am 19. September 2013) 
Bei den ausländischen Studierenden in Wien zeigt sich, dass das räumliche Einzugsgebiet einerseits sehr breit, andererseits hochgradig konzentriert ist. Der überwiegende Teil, vier von fünf ausländischen Studierenden, kommt aus Europa ${ }^{2}$, wobei die Schätzung zeigt, dass die westeuropäischen Staaten am bedeutendsten sind (vgl. Tabelle 5.5). Die Internationalisierung des Hochschulstandortes ist also im Wesentlichen eine Europäisierung. Außerhalb Europas ist Asien mit 14,1 \% der ausländischen Studierenden (insbesondere Westasien) die wichtigste Herkunftsregion, wobei die Zuordnung der Türkei zu dieser Region hier eine zentrale Variable ist. Die „restlichen“ Weltregionen Afrika, Amerika, Ozeanien und das restliche Asien - machen zusammen gerade einmal $10,1 \%$, in absoluten Zahlen 4.724, ausländische Studierende aus.

Zwischen den Hochschultypen lassen sich - wie auch beim Niveau der Internationalisierung - durchaus Unterschiede hinsichtlich der Herkunft der ausländischen Studierenden feststellen (vgl. Abbildung 5.3): Auf europäischer Ebene stammen bei den Universitäten knapp die Hälfte der Europäer aus dem westlichen Europa (49,0 \%), bei den Fachhochschulen hingegen knapp zwei Drittel (65,2 \%), bei den Privatuniversitäten hingegen nur ein Drittel, während hier die Studierenden aus Südeuropa mit 39,1 \%

Abbildung 5.3: Herkunft der ausländischen Studierenden Wiens nach Regionen innerhalb Europas für alle Hochschulen (WS 2011)

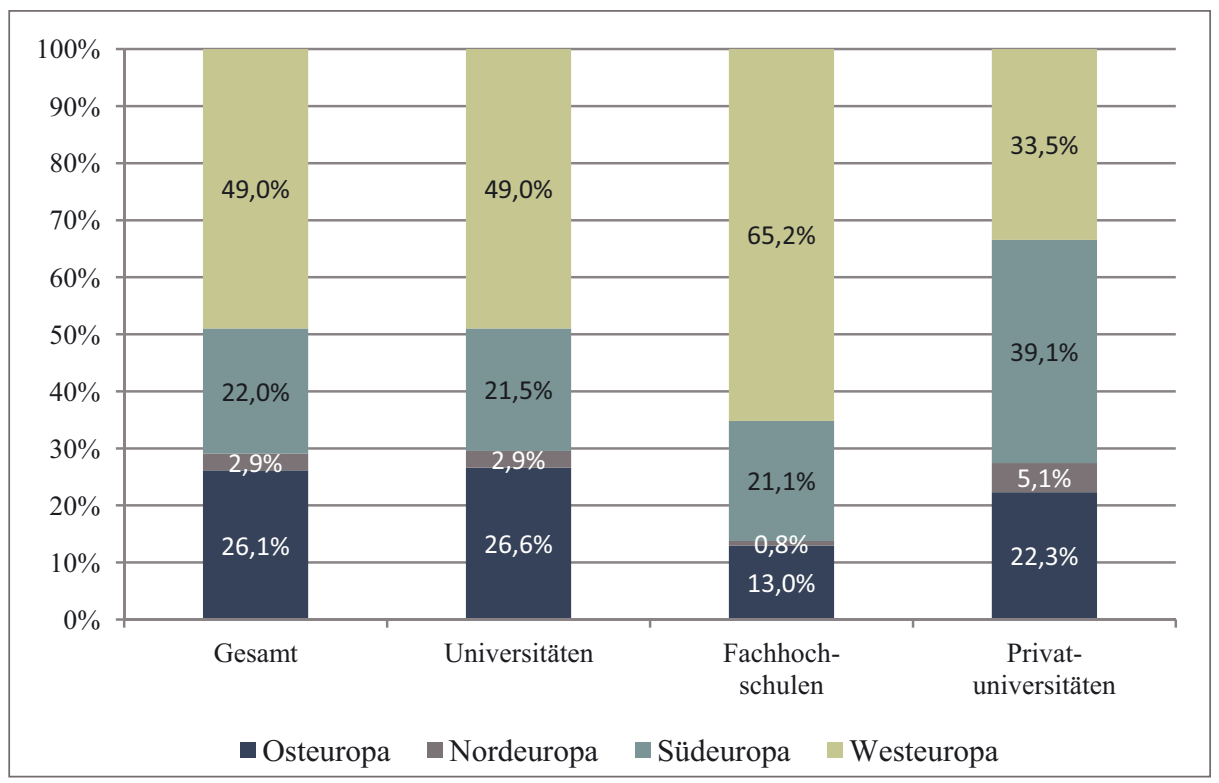

Quellen: eigene Erhebung 2013; uni:data 2013; eigene Berechnungen.

2 In dieser Studie erfolgt die regionale Zuteilung der Länder nach Großregionen/Kontinenten gemäß der Empfehlung der Vereinten Nationen. Aus diesem Grund wird beispielsweise die Türkei nicht zu Europa, sondern zu Asien gezählt (Westasien). 
die größte Gruppe darstellen. Der Anteil osteuropäischer Studierender ist an den Universitäten am höchsten, was vermutlich auch ein Effekt der niedrigen Zugangsbeschränkungen ist. Auf außereuropäischer, globaler Ebene unterscheiden sich die Gewichte der Herkunftsregionen zwischen Universitäten und Privatuniversitäten kaum (vgl. Abbildung 5.4), nur bei den Fachhochschulen dominiert Asien, insbesondere Westasien und die Türkei, besonders stark (83,3\%), während Studierende aus Afrika und Ozeanien überhaupt nicht vertreten sind.

\section{Abbildung 5.4: Herkunft der ausländischen Studierenden Wiens nach Kontinenten (ohne} Europa) für alle Hochschulen (WS 2011)

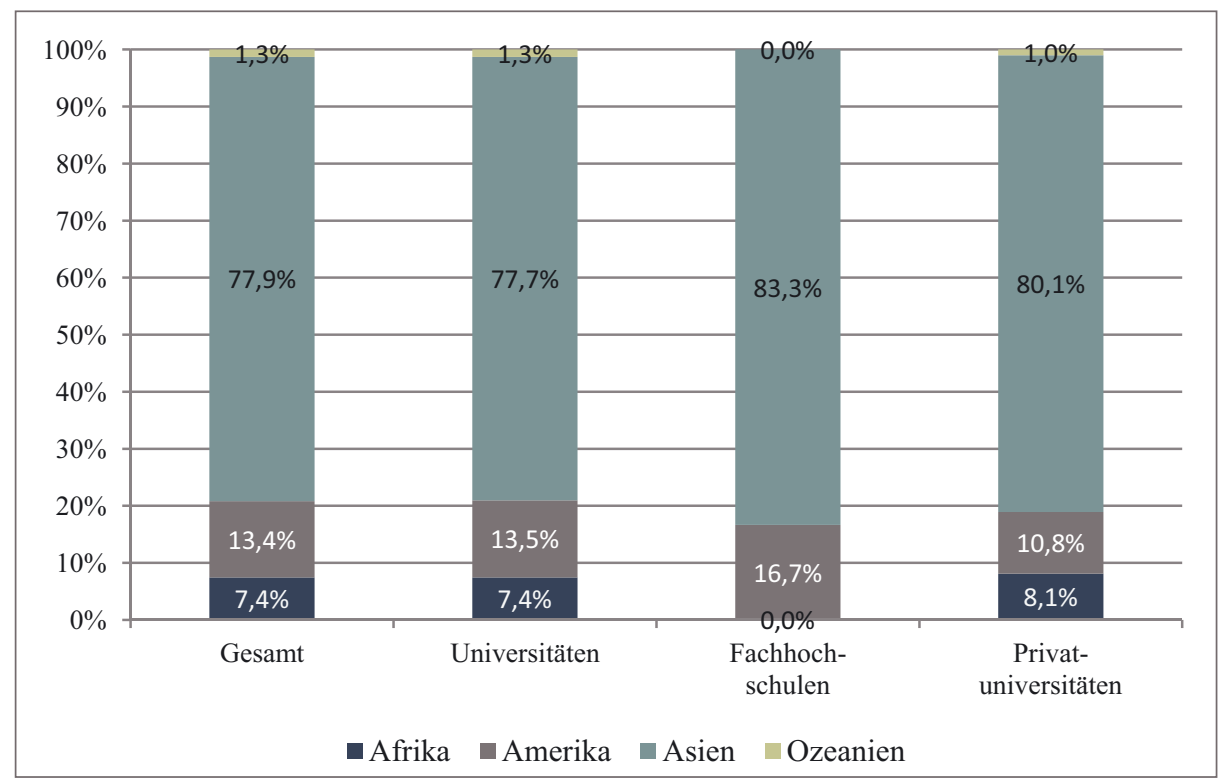

Quellen: eigene Erhebung 2013; uni:data 2013; eigene Berechnungen.

Wie die Herkunft der ausländischen Studierenden an Wiener Hochschulen konzentriert ist, lässt sich auf Ebene der Staaten präziser darstellen. Tabelle 5.6 zeigt die 20 wichtigsten Herkunftsländer, allerdings nur für jene Hochschulen, die dazu Angaben gemacht haben (vgl. Anhang I); auf eine Hochrechnung der anderen Hochschulen wurde aufgrund der beträchtlichen Unschärfen bei einer Schätzung auf Länderebene in diesem Fall verzichtet. An diesen elf Hochschulen entfallen in Summe 84,2 \% der ausländischen Studierenden auf die 20 wichtigsten Herkunftsländer. Das mit Abstand wichtigste Herkunftsland ist Deutschland, von wo mehr als jeder Dritte ausländische Studierende (35,8 \%) stammt. Die Bedeutung der gemeinsamen Sprache (neben den Zugangsberechtigungen) unterstreicht auch Italien, das aufgrund der zahlreichen Südtiroler Studierenden in Wien auf Rang 3 liegt (5,4\%). An zweiter Stelle steht hingegen 
die Türkei mit 6,9\%, weiters Bosnien und Herzegowina (3,8 \%); es handelt sich dabei um Staaten, die durch eine lange Tradition der Gastarbeitermigration mit Österreich verbunden sind. In diesem Zusammenhang sind auch Serbien (Rang 9, 3,0 \%) und Kroatien (Rang 11, 2,4 \%) zu nennen. Hier ist aber anzumerken, dass die Statistik nicht unterscheidet, ob es sich bei den Studierenden um Personen handelt, die zum Studium nach Wien gekommen sind, oder um in Österreich lebende bzw. geborene Ausländer. Bemerkenswert ist weiter, dass sich zusätzlich zu den drei genannten Westbalkanstaaten unter den Top-20-Ländern noch weitere neun osteuropäische Länder finden, womit die Bedeutung Ost- und Südosteuropas als Herkunftsregion für ausländische Studie-

Tabelle 5.6: Die 20 wichtigsten Herkunftsländer ausländischer Studierenden (WS 2009 bis WS 2011) für an der Erhebung teilnehmenden Hochschulen

\begin{tabular}{|c|c|c|c|c|c|}
\hline Rang 2011 & Herkunftsland & 2009 & 2010 & 2011 & $\begin{array}{c}\text { Anteil } 2011 \\
(\%)\end{array}$ \\
\hline 1 & Deutschland & 9.761 & 11.008 & 12.115 & 35,8 \\
\hline 2 & Türkei & 1.934 & 2.176 & 2.330 & 6,9 \\
\hline 3 & Italien & 1.697 & 1.819 & 1.838 & 5,4 \\
\hline 4 & Bosnien und Herzegowina & 1.310 & 1.254 & 1.289 & 3,8 \\
\hline 5 & Slowakei & 1.200 & 1.243 & 1.199 & 3,5 \\
\hline 6 & Bulgarien & 913 & 1.033 & 1.108 & 3,3 \\
\hline 7 & Polen & 1.132 & 1.122 & 1.087 & 3,2 \\
\hline 8 & Serbien & 893 & 952 & 1.017 & 3,0 \\
\hline 9 & Rumänien & 733 & 864 & 971 & 2,9 \\
\hline 10 & Ungarn & 866 & 964 & 958 & 2,8 \\
\hline 11 & Kroatien & 723 & 794 & 811 & 2,4 \\
\hline 12 & Ukraine & 524 & 569 & 657 & 1,9 \\
\hline 13 & Russische Föderation & 408 & 493 & 597 & 1,8 \\
\hline 14 & China & 559 & 497 & 452 & 1,3 \\
\hline 15 & Tschechische Republik & 378 & 381 & 416 & 1,2 \\
\hline 16 & Iran & 328 & 352 & 407 & 1,2 \\
\hline 17 & Luxemburg & 258 & 301 & 337 & 1,0 \\
\hline 18 & Vereinigte Staaten & 263 & 282 & 319 & 0,9 \\
\hline 19 & Frankreich & 292 & 310 & 308 & 0,9 \\
\hline \multirow[t]{3}{*}{20} & Slowenien & 278 & 300 & 303 & 0,9 \\
\hline & Top 20 & 24.450 & 26.714 & 28.519 & 84,2 \\
\hline & Gesamt & 29.068 & 31.651 & 33.872 & 100,0 \\
\hline
\end{tabular}

Quelle: eigene Erhebung 2013. 
Karte 5.5: Herkunft der ausländischen Studierenden Wiens (WS 2011) innerhalb Europas

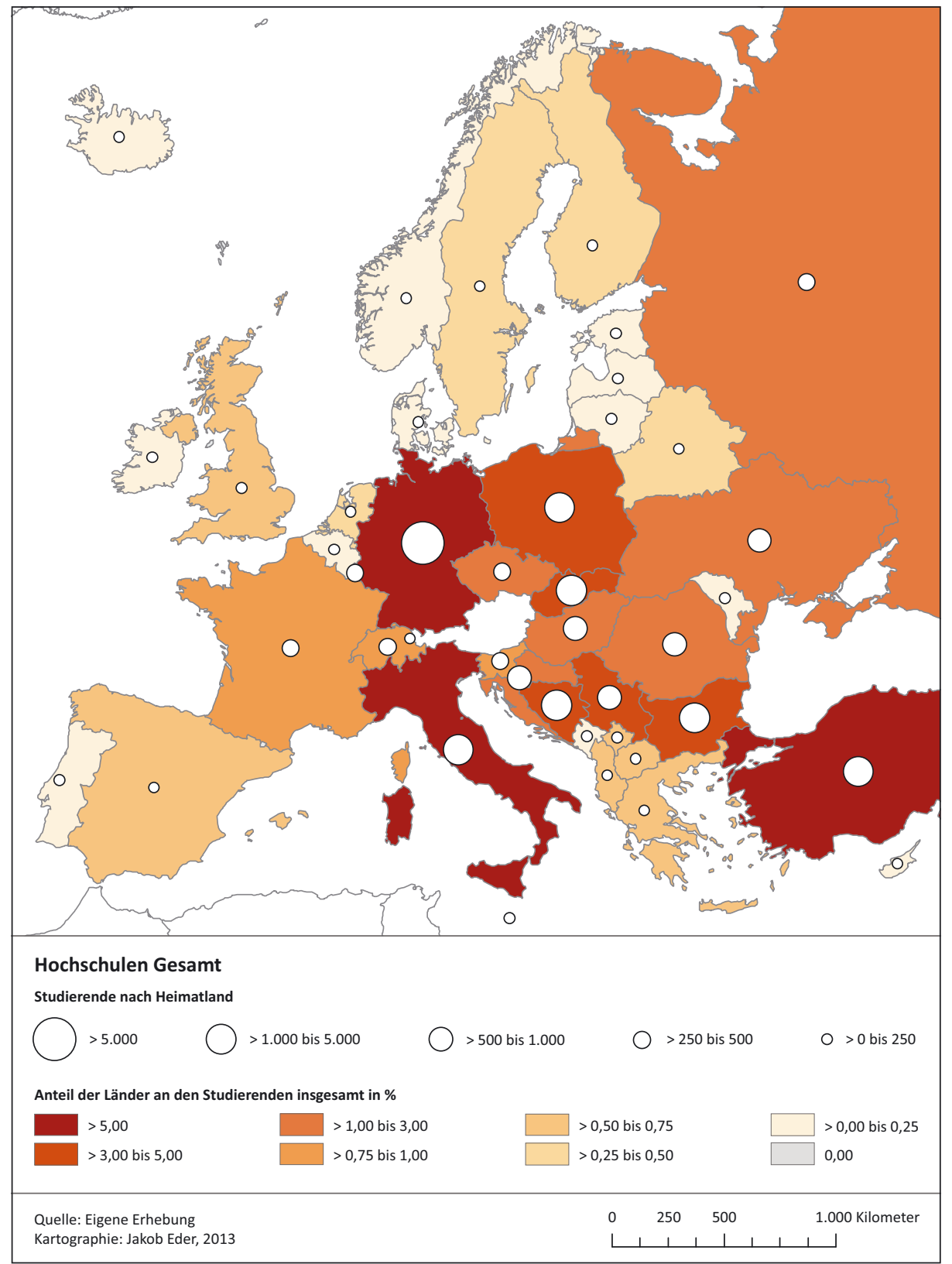


Karte 5.6: Weltweite Herkunft der ausländischen Studierenden Wiens (WS 2011)

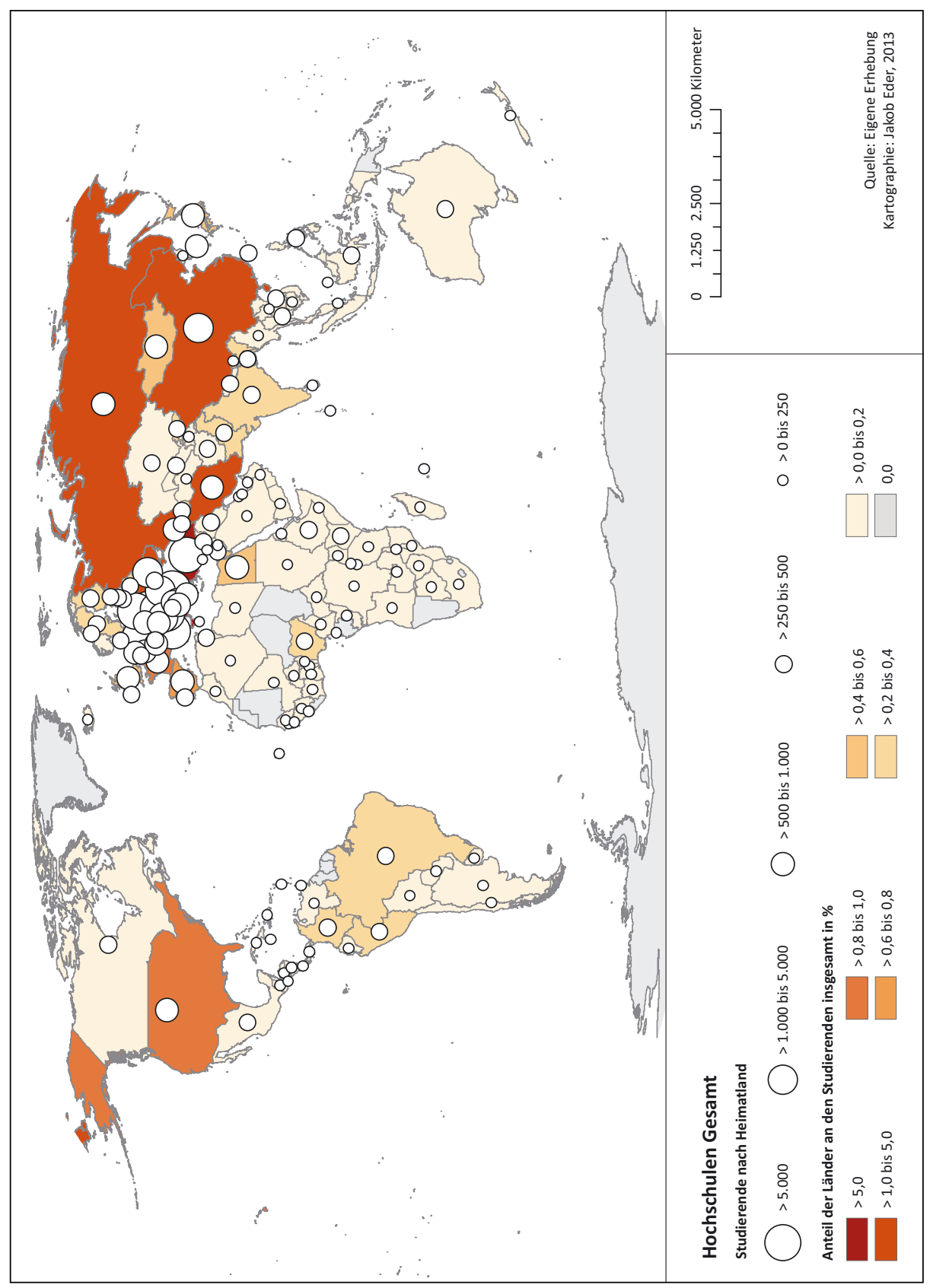


rende unterstrichen wird. China und Iran auf den Rängen 14 und 16 sind neben der Türkei weitere außereuropäische Herkunftsregionen, aus denen jedoch ebenfalls Einwanderergruppen in Wien existieren. Die Vereinigten Staaten auf Rang 18 mit 0,9 \% (319) der ausländischen Studierenden, sind das erste außereuropäische Land, das keine nennenswerte Einwanderer-Community in Wien besitzt.

Westeuropäische Staaten haben als Herkunftsland für ausländische Studierende eine geringe Bedeutung, wenn man das deutschsprachige bzw. sprachverwandte (Luxemburg) Ausland nicht berücksichtigt. Frankreich etwa liegt mit gerade 308 Studierenden nur auf Rang 19, während wichtige Wissenschaftsnationen wie Großbritannien (Rang 29) oder die Niederlande (Rang 35) keine Rolle spielen. Auch für die Schweiz (Rang 23) scheint, trotz der räumlichen Nähe und gemeinsamen Sprache, Wien kein attraktiver Studienort zu sein.

Die Internationalisierung des Hochschulstandortes Wien speist sich somit aus drei Quellen: erstens Studierenden aus Deutschland und Italien/Südtirol, die aufgrund des leichteren Hochschulzuganges und der sprachlich-kulturellen Nähe in Wien studieren; zweitens der traditionellen Verbindung zu den ehemaligen Gastarbeiterländern Türkei und den Staaten des Westbalkans und drittens der starken Anziehungskraft für Studierende aus dem östlichen Europa. Überlagert wird dieses historisch-kulturelle Grundmuster durch das spezifische Angebot der Hochschulen (vgl. Tabelle 5.7) sowie durch die schon angesprochene Hierarchie der europäischen Hochschullandschaft: Studierende der Universität Wien zieht es im ERASMUS-Programm in das westliche Europa, umgekehrt besitzt Wien eine starke Anziehung auf Studierende aus den Staaten des östlichen Europas (vgl. Universität Wien 2011, 11 f.; Meyer et al. 2012).

Tabelle 5.7: Die drei wichtigsten Herkunftsländer ausländischer Studierender (WS 2011) nach Hochschulen

\begin{tabular}{lcccc}
\hline \multicolumn{1}{c}{ Hochschule } & Rang 1 & Rang 2 & Rang 3 & $\begin{array}{c}\text { Anteil Top 3 } \\
(\%)\end{array}$ \\
\hline Universität Wien & Deutschland & Türkei & Italien & 48,7 \\
Universität für Bodenkultur & Deutschland & Italien & Türkei & 55,5 \\
Veterinärmedizinische Universität & Deutschland & Italien & Luxemburg & 81,2 \\
Wirtschaftsuniversität Wien & Deutschland & Bulgarien & Slowakei & 46,2 \\
Akademie der bildenden Künste & Deutschland & Italien & Dänemark & 50,9 \\
\hline Fachhochschule Campus Wien & Deutschland & Italien & Schweiz & 91,7 \\
Ferdinand Porsche FernFH & Deutschland & Polen & - & 100,0 \\
FH Wien der WK Wien & Deutschland & Italien & Kroatien & 67,8 \\
\hline Modul University & Kroatien & Deutschland & Russland & 22,5 \\
Privatuniversität Konservatorium & Deutschland & Japan & Südkorea & 37,3 \\
Sigmund Freud Privatuniversität & Slowenien & Deutschland & Italien & 66,8 \\
\hline
\end{tabular}

Quelle: eigene Erhebung 2013. 


\subsection{Alters- und Geschlechtsstruktur der Studierenden}

Die Geschlechtsstruktur an den Wiener Hochschulen zeigt in Summe einen leichten Überhang weiblicher Studierender: Bei den insgesamt 184.755 Studierenden im WS 2011 liegt der Frauenanteil bei 53,1 \%, wobei es zwischen den Einrichtungen beträchtliche Schwankungen gibt, die vom Studienangebot der Universitäten geprägt sind. An Hochschulen mit überwiegend technischen Studienfächern liegt der Anteil weiblicher Studierender weit unter der 50-\%-Marke (Technische Universität Wien 26,4 \%, FH Technikum 13,7 \%, Porsche FernFH 37,2 \%), an anderen Einrichtungen hingegen deutlich darüber, etwa an der Veterinärmedizinischen Universität $(81,0 \%)$, der Sigmund Freud Privatuniversität (71,6 \%) oder der Universität Wien (63,3 \%, vgl. Tabelle 5.8).

Das Verhältnis zwischen weiblichen und männlichen Studierenden schwankt nicht nur zwischen den Studienrichtungen, sondern auch nach dem Alter; in Summe ist im „regulären Studierendenalter“ der Überhang weiblicher Studierender am stärksten ausgeprägt und beläuft sich auf 55,9 \% an allen Hochschulen Wiens. Die Aufschlüsselung der Bevölkerungs- und Altersstruktur in sogenannten „Bevökerungspyramiden“ (Abbildung 5.5 und 5.6 sowie für einzelne Hochschulen im Anhang III) zeigt, dass mit zunehmendem Alter der Anteil weiblicher Studierender sukzessive abnimmt; schon ab der

Abbildung 5.5: Alters- und Geschlechtsstruktur der Studierenden an den Wiener Universitäten (WS 2011)

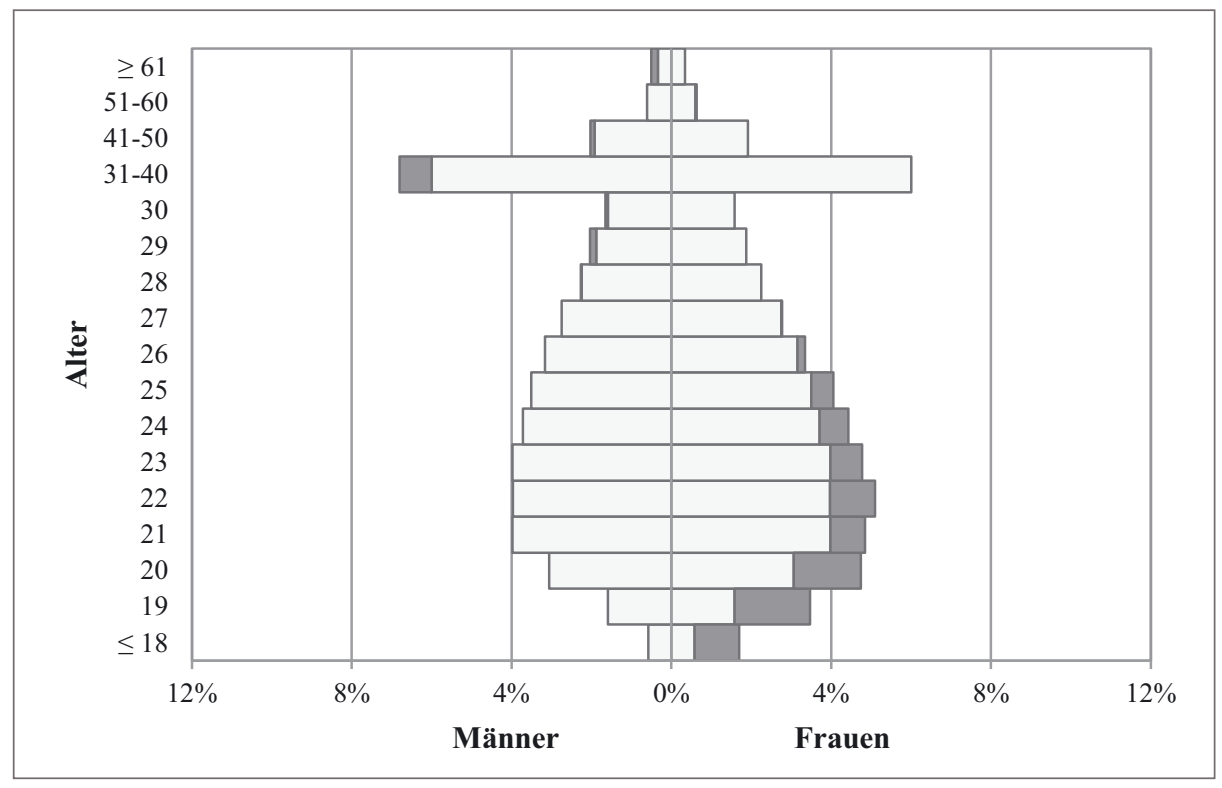

Quellen: eigene Erhebung 2013; uni:data 2013; eigene Berechnungen. 
Kohorte der 28-Jährigen besteht an den Wiener Universitäten ein schwacher Überhang an männlichen Studierenden. Ursache dafür dürfte eine geringfügig stärkere Nachfrage nach berufsbegleitendem Studium bei Männern sein; gleiches gilt für Studierende im Pensionsalter. Im Fall den Wiener Fachhochschulen ist der beschriebene Zusammenhang zwischen Alters- und Geschlechterproportion noch stärker ausgeprägt, was auf die hohe Zahl der berufsbegleitend Studierenden (siehe den hohen Anteil männlicher FH-Studierender bei den 31- bis 40-Jährigen, Abbildung 5.6) und nicht zuletzt auf das explizit berufsbegleitende Studienangebot zurückzuführen ist.

Tabelle 5.8: Alters- und Geschlechtsstruktur der Studierenden an den Hochschulen Wiens (WS 2011)

\begin{tabular}{lccc}
\hline \multicolumn{1}{c}{ Hochschule } & $\begin{array}{c}\text { Anteil der } \\
\text { weiblichen } \\
\text { Studierenden (\%) }\end{array}$ & $\begin{array}{c}\text { Anteil der } \\
\text { 19-26-Jährigen } \\
(\%)\end{array}$ & $\begin{array}{c}\text { Anteil der weiblichen } \\
\text { Studierenden an den } \\
\text { 19-26-Jährigen (\%) }\end{array}$ \\
\hline Universität Wien & 63,3 & 60,3 & 66,4 \\
Medizinische Universität Wien & 49,3 & 61,3 & 52,0 \\
Technische Universität Wien & 26,4 & 59,8 & 28,6 \\
Universität für Bodenkultur Wien & 47,3 & 60,0 & 52,0 \\
Veterinärmedizinische Universität Wien & 81,0 & 58,0 & 83,3 \\
Wirtschaftsuniversität Wien & 48,1 & 70,3 & 49,8 \\
Universität f. angewandte Kunst Wien & 58,6 & 65,1 & 56,9 \\
Univ. f. Musik u. darstellende Kunst Wien & 55,4 & 65,2 & 53,7 \\
Akademie der bildenden Künste Wien & 61,6 & 64,9 & 59,9 \\
Universitäten gesamt & $\mathbf{5 3 , 7}$ & $\mathbf{6 1 , 8}$ & $\mathbf{5 6 , 2}$ \\
\hline Fachhochschule Campus Wien & 54,4 & 59,0 & 61,9 \\
Fachhochschule des bfi Wien GmbH & 45,3 & 57,4 & 52,9 \\
Fachhochschule Technikum Wien & 13,7 & 51,8 & 17,7 \\
Ferdinand Porsche FernFH & 37,2 & 15,8 & 42,9 \\
FH Wien der WK Wien & 58,3 & 68,2 & 63,0 \\
Fachhochschulen gesamt & $\mathbf{4 1 , 7}$ & $\mathbf{5 6 , 8}$ & $\mathbf{4 9 , 3}$ \\
\hline Modul University Vienna & 59,7 & 71,1 & 62,7 \\
Privatuniversität Konservatorium Wien & 56,4 & 65,2 & 54,6 \\
Sigmund Freud Privatuniversität Wien & 71,6 & 41,5 & 75,8 \\
Webster University Wien & 50,2 & 61,5 & 52,7 \\
Privatuniversitäten gesamt & $\mathbf{6 1 , 4}$ & $\mathbf{5 6 , 3}$ & $\mathbf{6 1 , 5}$ \\
\hline \hline Gesamt & $\mathbf{5 3 , 1}$ & $\mathbf{6 1 , 4}$ & $\mathbf{5 5 , 9}$ \\
\hline
\end{tabular}

Quellen: eigene Erhebung 2013; uni:data 2013; eigene Berechnungen. 
Abbildung 5.6: Alters- und Geschlechtsstruktur der Studierenden an den Wiener Fachhochschulen (WS 2011)

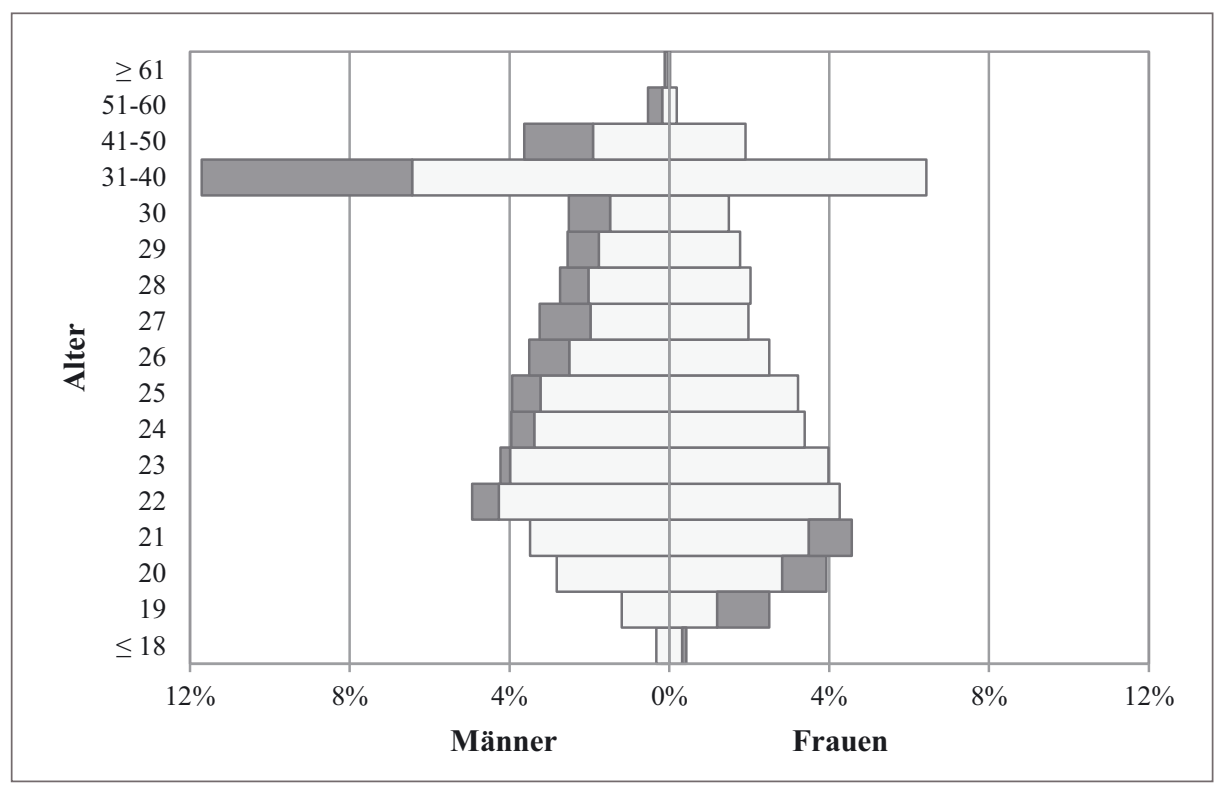

Quellen: eigene Erhebung 2013; uni:data 2013; eigene Berechnungen.

Auf die Bedeutung der Wiener Hochschulen für die Bevölkerung Wien wurde schon hingewiesen, die Studierenden machen immerhin 10,7 \% der Gesamtbevölkerung aus, nach Tagespendlern aus dem Wiener Umland bereinigt sind es 9,1 \% (vgl. Tabelle 5.4). Berücksichtigt man hingegen die Altersstruktur der Studierenden und setzt man diese mit der Bevölkerung der gleichen Kohorte in ein Verhältnis, so verschieben sich die Relationen deutlich (vgl. Tabelle 5.9): So stehen den 193.614 Einwohnern Wiens in der Alterskohorte der 19- bis 26-Jährigen immerhin 112.455 Studierende gegenüber,

Tabelle 5.9: Verhältnis Studierende/Gesamtbevölkerung der Wiener Hochschulen zur Stadt Wien (WS 2011) für die Alterskohorte der 19-26-Jährigen

\begin{tabular}{|c|c|c|c|c|c|}
\hline & \multirow{3}{*}{$\begin{array}{l}\text { Bevölkerung Wien } \\
\text { (19- bis 26-Jährige) }\end{array}$} & \multicolumn{4}{|c|}{ Studierende an Wiener Hochschulen (19- bis 26-Jährige) } \\
\hline & & \multicolumn{2}{|c|}{ nicht bereinigt } & \multicolumn{2}{|c|}{ bereinigt } \\
\hline & & absolut & in $\%$ & absolut & in $\%$ \\
\hline Gesamt & 193.614 & 112.455 & 58,1 & 95.699 & 49,4 \\
\hline Frauen & 99.101 & 55.839 & 56,3 & 47.519 & 47,9 \\
\hline Männer & 94.513 & 56.617 & 59,9 & 48.181 & 51,0 \\
\hline
\end{tabular}

Quellen: eigene Erhebung 2013; Uni-data 2013; Stadt Wien 2013; eigene Berechnungen. 
was einen Anteil von 58,1\% der Gesamtbevölkerung in dieser Altersgruppe ausmacht. Selbst wenn man die Studierendenzahl um die 14,9\% der Tagespendler aus dem Wiener Umland bereinigt (wobei eine konstante Pendlerquote für alle Alterskohorten anzunehmen ist), handelt es sich noch immer um 95.699 Studierende, die knapp die Hälfte (49,4 \%) der jeweiligen Alterskohorte ausmachen, bei den männlichen Studierenden (bereinigt) liegt der Anteil an der Gesamtbevölkerung sogar über der Hälfte. 